unresolved. Is it some property inherent to the swinging gate or to the rest of the colicin molecule? It will be interesting to see whether other domains, even large proteins, can be engineered into this region and flipped across the membrane.

It is not understood how channels such as the potassium channel or cystic fibrosis transmembrane regulator are opened or closed by binding of small molecules or by covalent modification. Let's assume that the physical properties of some domains of the ion channel allow them to bob in and out of the membrane reversibly. Modification of this domain, like the binding of streptavidin to colicin, would then trap the channel in an open or closed configuration. The modification does not induce a conformational change but instead stabilizes changes driven by brownian motion. Thirty years ago it was proposed that brownian motion could drive a machine if given a gradient of thermal (chemical) energy ${ }^{12}$. This model may be applicable to ion channels, where thermal motions drive their opening and closing and the chemical energy of modification locks them into one state or another. Such brownian ratchets ${ }^{13}$ have been used to describe protein translocation across membranes ${ }^{14}$, filapodial extension and the movement of bacteria and motor molecules ${ }^{15}$.

The results of Slatin et al. ${ }^{1}$ will certainly spur a rethinking among colicin toxicologists and channel physiologists. Together with demonstrations that other hydrophilic molecules such as proteins ${ }^{16}$ and nucleotides ${ }^{17}$ can also cross membranes through aqueous channels, this discovery should intrigue everyone interested in the functioning of membrane proteins.

Sanford Simon is in the Laboratory of Cellular Biophysics, Box 304, The Rockefeller University, 1230 York Avenue, New York, New York 10021, USA.

1. Slatin, S. L., Qiu, X.-Q., Jakes, K. S. \& Finkelstein, A. Nature 371, 158-161 (1994).

2. Duché, D. Parker. M. W., Gonzalez-Manas, J. M., Pattus, F \& Baty D f biol Chem. 269, 6332-6339 (1994).

3. Cramer, W. A. Cohen, F. S. Merrill, A. R. \& Song. H. Y Molec. Microbiol. 4, 519-526 (1990)

4. Baumann, G. \& Mueller, P.J. supramolec. Struct. 2 $538-557$ (1974).

5. Schindler, M. \& Rosenbusch. J.P. J. Cell Biol. 92 $742-746(1982)$

6. White, B. H. \& Cohen, J. B. Biochemistry 27, 8741 (1988)

7. Zimmerberg J \& Parsegian V A. Nature 323,36 (1986)

8. Lingappa, V. R. Katz, F. N., Lodish, H. F. \& Blobel, G. J. biol. Chem. 253, 8667-8670 (1978).

9. Friediander, M. \& Biobel, G. Nature 318, 338-343 (1985).

10. Ostapchuk, P.. Hearing, P. \& Ganem, D. EMBO J. 13 1048-1057 (1994)

11. Bruss, V.. Lu, X.. Thomssen, R. \& Gerlich, W. H. EMBO J. 13. 2273-2279 (1994)

12. Feynman, R. Leighton, R. \& Sands, M. The Feynman Feynman, R., Leighton, R. \& Sands, M. The Feyn
Lectures on Physics (Addison-Wesley, 1963).

13. Magnasco, M. Phys. Rev. Lett. 72, 2656-2659 (1994).

14. Simon, S. M., Peskin, C. S. \& Oster, G. F. Proc. natn. Acad. Sci. U.S.A. 89, 3770-3774 (1992)

15. Peskin, C. S., Odell, G. M. \& Oster, G. F. Biophys. J. 65 316-324 (1993).

16. Simon S. M \& Blobel G. Cell65, 371-380 (1991)

17. Boulanger, P. \& Letellier, L. J. biol. Chem. 267, 3168 3172 (1992)

\section{From the simple to the complex}

\section{P. N. Goodfellow and K. Schmitt}

THE genes that cause Duchenne muscular dystrophy, Huntington's chorea, adult polycystic kidney disease and cystic fibrosis, to name a few, have all been identified by a combination of classical genetics and molecular biology. These diseases are genetically simple and relatively rare, whereas diseases such as diabetes that impose the largest burdens on Western societies are very common and often genetically complex. Unravelling the aetiology of complex genetic diseases used to be an intractable problem, but recent advances in high-throughput genotyping and high-resolution genetic mapping should lift the gloomy prognosis. As described on pages $130^{1}$ and $161^{2}$ of this issue, two groups have searched the entire genome to identify new genetic components of a complex genetic form of diabetes known as type 1 insulindependent diabetes mellitus (IDDM; see box).

A simple genetic disease can be described by a slight modification of Beadle and Tatum's famous dictum: one gene, one polypeptide, one disease; the inheritance of such diseases follows the laws of mendelian genetics. A complex genetic disease shows no discernible inheritance pattern but has a tendency to cluster in families. Family members share most of their genes but also their environment: both factors can cause familial clustering of a disease - an issue endlessly debated as nature versus nurture. For each disease, the problem can be reduced to how many genes are involved and what, if any, the environmental factors are. The amount of familial clustering of a disease can be quantified by comparing the increased disease risk in a sibling of a patient with the risk in the population as a whole. Comparing the difference in risk between identical twins and dizygotic twins can give an indication of the genetic contribution to this increased disease risk, leaving the problem of identifying the genes involved and measuring the relative contributions of each gene. For inbred animals and plants, in which both the starting genotypes and phenotypes are well defined, several methods have been successful ${ }^{3,4}$.

But human genetics demands a different approach. Affected members of a family will share the genes causing the disease and this sharing will manifest itself as an excess of sharing over the $50 \%$ expected at random in siblings 5 . Disease genes can be identified by co-segregation with linked genetic markers which will show identity by descent in affected individuals. A genome scan combined with such an analysis makes no a priori assumptions about the number of genes involved in a disease, nor the types of interaction between them. The markers used must be close enough that recombination with the disease locus is unlikely and sharing among individuals can only be tested if the markers are polymorphic. The microsatellite markers developed at Généthon and elsewhere are ideal for this purpose: they are highly polymorphic and sufficient numbers of them have been positioned to provide a good coverage of the complete genome ${ }^{6}$.

Nevertheless, the experiments are far from trivial. Hundreds of families containing more than one affected offspring must be found. The more families tested, the smaller is the genetic contribution to the disease that can be revealed. All individuals must be clinically evaluated, their DNA isolated and then tested. A collection of one hundred families requires over 120,000 typings to complete a gene scan of

\title{
A brief history of diabetes
}

DIABETES mellitus was recognized long ago in ancient Egypt but it was not until the last century that investigation began into the biochemical features of the disease, eventually revealing the association between insulin and blood glucose levels that is now well known. There are two types of diabetes, insulin-dependent and non-insulin-dependent diabetes mellitus (IDDM or type 1, and NIDDM or type 2, respectively). IDDM usually affects children and results from an autoimmune response against the pancreas which causes selective destruction of insulin-producing cells and failure of insulin secretion in response to increased blood glucose. As insulin controls the uptake of glucose into cells, this lack of hormone forces them to switch to alternative fuels, with life-threatening metabolic consequences. Lifelong administration of insulin is essential in this condition.

Evidence for the involvement of genetic factors came from animal models (the NOD mouse and BB rat) and the increased risk of developing IDDM for members of a family affected with the disease. Population studies have shown that there is an association of IDDM with certain genes (DR3 and DR4) in the major histocompatibility complex, and other gene associations have now been identified. On the other hand, NIDDM affects adults and is usually due to a combination of impaired insulin secretion and insensitivity of the target tissue to the hormone. These two forms of diabetes affect between 2 and 4 percent of the UK population.

P.N.G.\& K.S. 